\title{
PROCESS AND SYSTEMS Making the case for spirometry as part of the perioperative multidisciplinary team assessment
}

\author{
Authors: Thomas Chambers, ${ }^{\mathrm{A}}$ Mevan Gooneratne, ${ }^{\mathrm{B}}$ Richa Singh, ${ }^{\mathrm{C}}$ Ching Pang, ${ }^{\mathrm{B}}$ Gayle McDonnell ${ }^{\mathrm{D}}$ \\ and William Ricketts ${ }^{\mathrm{C}}$
}

\begin{abstract}
Chronic obstructive pulmonary disease (COPD) is a leading cause of morbidity and mortality and is significantly underdiagnosed in the community. Respiratory impairment is a key risk factor for perioperative morbidity and mortality.

The National Institute for Health and Care Excellence (NICE) does not recommend routine spirometry before major surgery. However, in this article, we present the potential benefits of targeted spirometry in high-risk patient groups.

Of 183 patients who underwent targeted preoperative spirometry, $25 / 70$ (35.7\%) of those with airflow obstruction had no previously known respiratory diagnosis. Of patients with known COPD, $20 / 46$ (43.5\%) were not prescribed optimum inhaled therapies for their degree of lung function deficit.

Knowledge of lung function in respiratory disease helps to optimise patients perioperatively and facilitate shared decision making regarding the benefits and risk of surgeries. We propose that targeted spirometry should be used as part of the perioperative multidisciplinary team assessment of selected patients.
\end{abstract}

KEYWORDS: spirometry, perioperative medicine, COPD, airflow obstruction, shared decision making

DOI: 10.7861/fhj.2021-0116

\section{Introduction}

Chronic obstructive pulmonary disease (COPD) causes significant global morbidity and mortality. The World Health Organization (WHO) report that, behind ischaemic heart disease and stroke, it is the third leading cause of death globally. ${ }^{1}$

Despite this, COPD is significantly underdiagnosed. A 2018 Canadian study found that in a randomly selected population of 1,403 adults $>40$ years old, $13.7 \%$ of individuals had undiagnosed

Authors: ${ }^{\text {A }}$ linical fellow, Barts Health NHS Trust, London, UK; ${ }^{B}$ consultant anaesthetist, Barts Health NHS Trust, London UK: ${ }^{C}$ consultant respiratory physician, Barts Health NHS Trust, London,

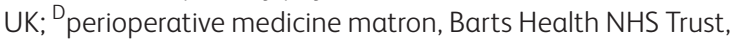
London, UK
COPD. ${ }^{2}$ In 2020, a UK community-based screening programme found that, of ever-smokers aged 55-74 years with airflow obstruction, $49.7 \%$ had no previous COPD diagnosis. ${ }^{3}$

COPD increases the risk of a raft of perioperative complications, particularly postoperative respiratory failure. ${ }^{4}$ Indeed, a history of COPD is a key parameter in perioperative morbidity and mortality prediction tools such as POSSUM and P-POSSUM. ${ }^{5}$ In light of this, detection of undiagnosed COPD to facilitate preoperative optimisation, with a view to reducing postoperative complications, would seem a worthwhile strategy.

The National Institute for Health and Care Excellence (NICE) suggests a senior anaesthetist should be consulted if a patient is the American Society of Anaesthesiologists' (ASA's) grade 3 or 4 due to suspected or confirmed respiratory disease. ${ }^{6}$ However, NICE specifically recommend that spirometry should not be performed routinely for elective major surgery. ${ }^{6}$

Here, we propose the benefits of targeted spirometry and a subsequent perioperative multidisciplinary team (MDT) review in a high-risk assessment (HRA) clinic. Patients are referred to this clinic by their surgical team if they have significant cardiac, respiratory, anaesthetic or airway concerns. Approximately 35 patients a week are reviewed in this clinic.

We sought to develop this pathway to identify possible undiagnosed COPD in a preoperative population. Through identification and optimisation of these patients, and of those with known airways disease, we aimed to reduce perioperative risk and improve shared decision-making discussions around risks and benefits of surgery.

\section{Solution}

The staff in our nurse-led HRA clinic at an east-London tertiary centre were trained in performing spirometry by respiratory physiologists. High-risk respiratory patients were invited for spirometry testing in the preoperative period. These patients include those undergoing major surgery (Fig 1) with known COPD or asthma but also current smokers or those aged $\geq 65$ years.

Spirometry was performed to assess forced expiratory volume in 1 second (FEV1) and forced vital capacity (FVC) in accordance with European Respiratory Society guidance using a Carefusion Microlab desktop standard spirometer. Values are expressed as absolute volumes and as a percentage of predicted values, based on sex, weight and height using standard Quanjer tables.

In this study, spirometry was performed without bronchodilators. This decision was made to reduce cost and time per test. This 


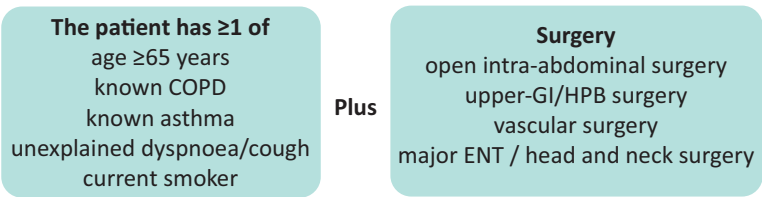

Fig 1. Criteria for spirometry within the high-risk assessment clinic. Patients were invited for spirometry if they were high risk for respiratory disease and were undergoing one of five types of major surgery. COPD = chronic obstructive pulmonary disease; ENT = ear, nose and throat; GI = gastrointestinal; $\mathrm{HPB}=$ hepatobiliary.

one-step process allows us to assess a larger volume of patients in a busy clinic. The spirometry acted as a severity assessment in those known to have COPD or asthma, and a screening test in those with no previous diagnosis. Once airflow obstruction is identified, patients can be referred for formal post-bronchodilator spirometry and a specialist respiratory review to diagnose obstructive airways disease.

COPD severity is assessed using data on exacerbation frequency and airflow obstruction as per NICE and the Global Initiative for Chronic Obstructive Lung Disease (GOLD). ${ }^{7.8}$

Patients with an FEV 1 of $50 \%$ predicted or more (ie mild or moderate COPD) are offered very brief advice (VBA) regarding smoking cessation, a referral to their local smoking cessation service, a chest $X$-ray, flu vaccination and inhaler technique review. ${ }^{9}$ Inhaled therapy is optimised as per trust guidance based on exacerbation frequency, Medical Research Council (MRC) breathlessness scores and severity of airflow obstruction. A pulmonary rehabilitation (PR) referral is also made if the MRC dyspnoea score is $\geq 3$.

In addition to the standard interventions offered to those with mild-moderate COPD, patients with newly identified airflow obstruction, severe COPD on spirometry (FEV $1<50 \%$ predicted), symptomatic asthma, cor pulmonale, haemoptysis or other clinical concerns receive a rapid referral to a dedicated MDT. The MDT comprises respiratory physicians, anaesthetists, geriatricians, cardiologists and specialist nurses. They are consulted for complex cases requiring further input on diagnosis, or specialist management of complex respiratory conditions. The MDT assess not only respiratory disease but other comorbidities. This holistic approach identifies surgical and anaesthetic options as well as opportunities for optimisation of COPD and other comorbidities, and frames discussion on the risk of delaying surgery to facilitate this.

\section{Outcome}

Between 06 February 2019 and 04 September 2020, 183 patients underwent spirometry. The mean age was 65.8 years (standard deviation (SD) 15) and $46 \%$ were men.

Out of 183 patients, 46/183 (25.1\%) had a previous COPD diagnosis. Of these patients known to have COPD, 18/46 (39.1\%) were current smokers. They were offered VBA on smoking cessation and a referral to their local smoking cessation clinic. ${ }^{9}$ Patients with an MRC dyspnoea score of three or more can be referred for PR. In our cohort, 16/46 (34.7\%) of patients fulfilled this criterion. Where safe to delay surgery, PR should be commenced for 8 weeks. Failing this, PR for $\geq 3$ weeks should be offered where possible.
SABA alone or no inhaled therapies for their COPD

On LAMA monotherapy when they should be on LAMA/LABA combination inhaler

\section{Other reasons}

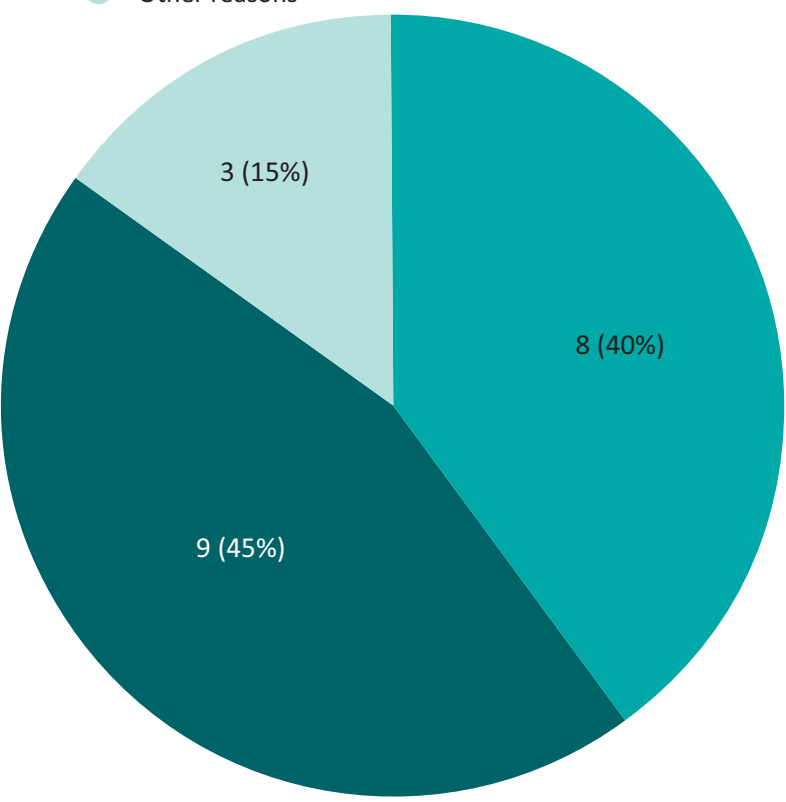

Fig 2. Areas for optimisation of inhaled therapies. 20/46 patients $(43.5 \%)$ had room for inhaler optimisation. Trust guidelines advise that patients should be on combined LABA/LAMA inhaler if Medical Research Council dyspnoea score $\geq 2$. COPD = chronic obstructive pulmonary disease: LABA = long-acting beta agonist; LAMA = long-acting muscarinic agonist; SABA $=$ short-acting beta agonist (eg salbutamol).

We found that $23 / 46$ (50\%) of patients with COPD were prescribed inhaled corticosteroids and 26/46 (56.5\%) patients were prescribed optimal inhaled therapy as per trust guidelines. Fig 2 highlights the areas for optimisation for the 20 patients not on optimal inhalers. These guidelines are based on GOLD recommendations and use symptoms, MRC breathlessness score, exacerbation frequency and spirometry to guide therapy. ${ }^{8}$ Exacerbation history was based on both self-reported community exacerbations (reviewing the patients' linked primary care records) and data on hospitalisations in the previous 12 months.

Of patients undergoing spirometry, 70/183 (38.2\%) had airflow obstruction (FEV1/FVC <0.7). In these patients, 25/70 (35.7\%) had no previous asthma or COPD diagnosis (Fig 3). Of the remaining patients, $105 / 183(57.3 \%)$ had normal spirometry and $8 / 183(4.4 \%)$ had evidence of restrictive lung disease with an FEV1 of $<80 \%$ predicted and FEV1/FVC $\geq 0.7$.

In our cohort of patients with newly identified obstructive spirometry, 14 patients had a smoking history and no respiratory background, suggesting possible undiagnosed COPD. In this group, 9/14 (64.3\%) were current smokers. As per guidelines, these patients require formal post-bronchodilator spirometry and a clinical review to diagnose airways disease. ${ }^{7,8}$ Of these patients, 9/14 (64.3\%) had an FEV1 <80\% predicted, suggesting at least moderate disease (FEV1\% predicted range $27.9 \%-122.4 \%$ ). 


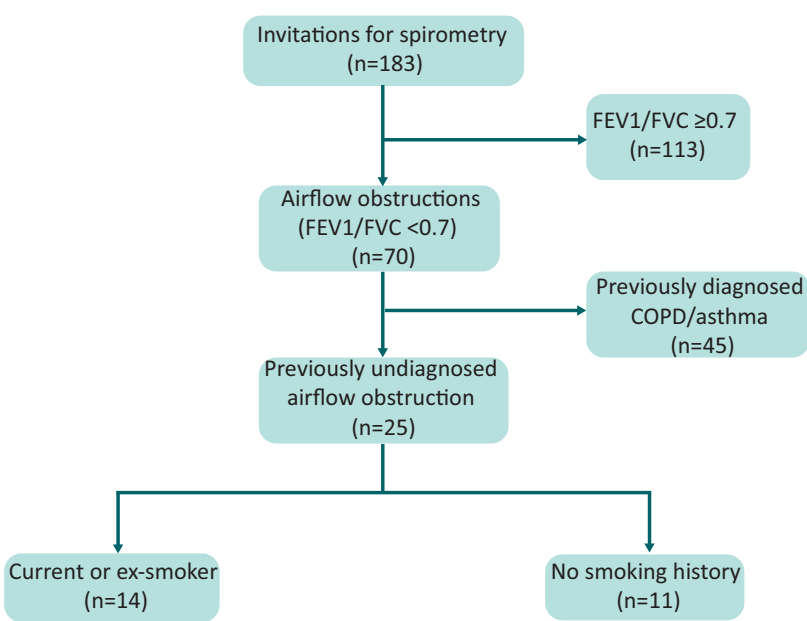

Fig 3. Spirometry results in those invited for testing. $\mathrm{COPD}=$ chronic obstructive pulmonary disease; FEV1 = forced expiratory volume in 1 second; $F V C=$ forced vital capacity.

\section{Conclusion and next steps}

Despite NICE not recommending preoperative spirometry, we propose that the preoperative period represents an opportunity to screen high-risk patients for airflow obstruction. ${ }^{6}$ Spirometry, combined with subsequent MDT review, is beneficial for a raft of reasons.

Firstly, it identifies possible undiagnosed COPD. We found $35.7 \%$ of patients with airflow obstruction had no previous respiratory diagnosis. This number is similar to a previous screening programme which identified nearly $49.7 \%$ of those with airflow obstruction had no previous COPD diagnosis. ${ }^{3}$ This work did not discount known patients with asthma which may explain the higher rate of 'unknown' airflow obstruction, especially if the patients had chronic asthma.

Secondly, up-to-date lung function and assessment facilitates MDT review and optimisation of patients with inhaled therapies and nonpharmacological interventions. More than $40 \%$ (20/46) of patients were not on optimum inhaled therapy as per trust guidance (Fig 2).

A sizable proportion of patients with airflow obstruction were current smokers (30/70; 42.9\%). For patients undergoing surgery, this time represents a 'teachable moment' for smoking cessation in which patients may be more open to discussions regarding the health benefits of smoking cessation. Smoking cessation is a key pillar of COPD treatment, and also reduces the risk of postoperative complications. ${ }^{7,8,10}$ As such, evidence-based VBA and smoking cessation clinic referral may be beneficial in both the pre- and postoperative period. ${ }^{9}$ Flu vaccination can also be offered to COPD patients during the winter months.

We identified that $35 \%(16 / 46)$ of patients with COPD had an MRC breathlessness score of $\geq 3$, making them suitable for PR. Prehabilitation for cancer surgery is recommended by Macmillan to improve cancer treatment outcomes and many recommendations are based on PR methodology. ${ }^{11}$ PR provides significant therapeutic benefits for patients with COPD in improving shortness of breath, exercise tolerance and quality of life. ${ }^{12,13}$ Data also suggest that intensive preoperative PR can achieve these benefits in a short period of time. ${ }^{12}$

Our work has a few key limitations. We did not have complete smoking status data for patients, such as pack-year history, or ex-smoker versus never-smoker status. We currently do not have post-MDT intervention data, including PR uptake, smoking cessation rates, optimisation of medications by primary care or perioperative complication rates. It would be useful to compare these with case-matched patients before spirometry testing was introduced in the HRA clinic. Furthermore, a formal analysis of whether this intervention results in a reduction of healthcare costs and improves quality-adjusted life years would be pertinent for developing this model further within both our trust and across other surgical centres. Formal patient feedback on the service is of key value to understand if patients felt more empowered and engaged with their medical care following review in this clinic.

However, focusing on perioperative care, our pathway can help stratify patients who are at high risk of perioperative complications. COPD is a key risk factor for morbidity and mortality, appearing in preoperative mortality scores such as POSSUM and P-POSSUM. ${ }^{5}$ It also predicts risk of postoperative respiratory failure and knowledge of lung function aids planning for appropriate levels of postoperative care. ${ }^{4}$

Finally, this pathway can enhance the process of shared decision making, a nuanced and important process in which clinicians and patients work together to make decisions about treatment. ${ }^{14}$ Knowledge of lung function can help contextualise the risks of morbidity and mortality. Using this as a platform facilitates patient engagement with these health decisions, which has shown to improve patient experience. ${ }^{15}$

\section{References}

1 World Health Organization. The top 10 causes of death. WHO, 2020. www.who.int/en/news-room/fact-sheets/detail/the-top-10causes-of-death [Accessed 28 June2021].

2 Gershon AS, Thiruchelvam D, Chapman KR et al. Health services burden of undiagnosed and overdiagnosed COPD. Chest 2018;153:1336-46

3 Balata H, Harvey ], Barber PV et al. Spirometry performed as part of the Manchester community-based lung cancer screening programme detects a high prevalence of airflow obstruction in individuals without a prior diagnosis of COPD. Thorax 2020;75:655-60.

4 Attaallah AF, Vallejo MC, Elzamzamy OM, Mueller MG, Eller WS. Perioperative risk factors for postoperative respiratory failure. J Perioper Pract 2019;29:49-53.

5 Prytherch DR, Whiteley MS, Higgins B et al. POSSUM and Portsmouth POSSUM for predicting mortality. Physiological and Operative Severity Score for the enUmeration of Mortality and morbidity. Br J Surg 1998;85:1217-20.

6 National Institute for Health and Care Excellence. Routine preoperative tests for elective surgery: NICE guideline [NG45]. NICE, 2016

7 National Institute for Health and Care Excellence. Chronic obstructive pulmonary disease in over 16s: diagnosis and management: NICE guideline [NG115]. NICE, 2019.

8 Global Initiative for Chronic Obstructive Lung Disease. Pocket guide to COPD diagnosis, management and prevention: a guide for healthcare professionals. GOLD, 2018:48.

9 National Institute for Health and Care Excellence. Stop smoking interventions and services: NICE guideline [NG92]. NICE, 2018

10 Thomsen T, Villebro N, Møller AM. Interventions for preoperative smoking cessation. Cochrane Database Syst Rev 2014;(3):CD002294.

11 Macmillan Cancer Support. Principles and guidance for prehabilitation within the management and support of people with cancer. Macmillan Cancer Support, 2020. www.macmillan.org.uk/ healthcare-professionals/news-and-resources/guides/principlesand-guidance-for-prehabilitation 
12 Cavalheri V, Granger C. Preoperative exercise training for patients with non-small cell lung cancer. Cochrane Database Syst Rev 2017;6:CD012020.

13 McCarthy B, Casey D, Devane D et al. Pulmonary rehabilitation for chronic obstructive pulmonary disease. Cochrane Database Syst Rev 2015;(2):CD003793.

14 Hester N, Pang C-L, Cho A, Kasivisvanathan R, Gooneratne M. Shared perioperative decision making: a shift in the doctor-patient paradigm. Br J Hosp Med 2019;80:216-9.
15 Santhirapala R, Moonesinghe R. Primum non nocere: is shared decision-making the answer? Perioper Med (Lond) 2016:5:16.

Address for correspondence: Dr Thomas Chambers, Respiratory Department, St Bartholomew's Hospital, West Smithfield, London EC1A 7BE, UK.

Email: thomas.chambers1@nhs.net

Twitter: @_TChambers 